\title{
MINERALOGIA E CRISTALOGRAFIA DA FRAÇÃO ARGILA DE HORIZONTES COESOS DE SOLOS NOS TABULEIROS COSTEIROS ${ }^{(1)}$
}

\author{
Neyde Fabíola Balarezo Giarola ${ }^{(2)}$, Herdjania Veras de Lima ${ }^{(3)}$, Ricardo \\ Espíndola Romero $^{(4)}$, André Maurício Brinatti ${ }^{(5)} \&$ Alvaro Pires da Silva $^{(6)}$
}

\begin{abstract}
RESUMO
A coesão de horizontes de solos do Brasil vem sendo associada, principalmente, a fatores físicos e químicos inter-relacionados, mas a influência das propriedades mineralógicas e cristalográficas dos minerais da fração argila também foi aventada por alguns pesquisadores. Neste trabalho, testou-se a hipótese de que a fração argila de horizontes coesos de solos do Sedimento Barreiras da faixa dos Tabuleiros Costeiros é dominantemente caulinítica e apresenta elevado grau de cristalinidade e ordenamento, o que pode favorecer $o$ ajuste face a face dos cristais. $O$ objetivo deste trabalho foi avaliar as características mineralógicas e cristalográficas dos minerais da fração argila de horizontes coesos provenientes de solos de sedimentos do Grupo Barreiras e uma possível contribuição para a ocorrência do caráter coeso. Para isso, foram analisados cinco horizontes coesos e um não-coeso de solos distribuídos ao longo da faixa dos Tabuleiros Costeiros. Também foi incluída, como referência, uma caulinita com elevado grau de cristalinidade. Todos os perfis foram analisados para classificação taxonômica e localização dos horizontes de interesse. Nas amostras dos horizontes selecionados, foram removidos a matéria orgânica e os óxidos. Após a dispersão de partículas, a fração argila foi individualizada, tratada e analisada por difração de raios X (DRX) para determinar os minerais e o grau de ordem/desordem estrutural, segundo o Método Especialista de Plançon \& Zacharie (1990). Todos os horizontes coesos apresentaram caulinitas
\end{abstract}

\footnotetext{
(1) Recebido para publicação em outubro de 2008 e aprovado em janeiro de 2009.

(2) Professor Adjunto do Departamento de Ciência do Solo e Engenharia Agrícola, Universidade Estadual de Ponta Grossa UEPG. Av. Gal. Carlos Cavalcanti 4748, CEP 84030-900 Ponta Grossa (PR). E-mail: neydef@uepg.br

(3) Professor Adjunto do Setor de Solos, Instituto de Ciências Agrárias, Universidade Federal Rural da Amazônia - UFRA. Av. Presidente Tancredo Neves 2501, CEP 66077-530 Belém (PA). E-mail: herdjania.lima@ufra.edu.br

(4) Professor Adjunto do Departamento de Ciência do Solo, Universidade Federal do Ceará - UFC. Av. Mister Hull s/n, Campus do Pici, Bloco 807, CEP 60455-760 Fortaleza (CE). E-mail: reromero@ufc.br

(5) Professor Adjunto do Departamento de Física, UEPG. E-mail: ambrinatti@gmail.com

(6) Professor Titular do Departamento de Ciência do Solo, Escola Superior de Agricultura "Luiz de Queiroz" - ESALQ/USP. Caixa Postal 09, Av. Pádua Dias 11, CEP 13418-900 Piracicaba (SP). Bolsista do CNPq. E-mail: apisilva@esalq.usp.br
} 
com grau de ordenamento estrutural similar ao do horizonte não-coeso e inferior à caulinita de referência, o que não permitiu associar o empacotamento da fração argila com a manifestação do caráter coeso nos horizontes estudados, a partir das avalioações obtidas pelo método utilizado.

Termos de indexação: caulinitas, horizontes adensados, propriedades cristalográficas, sistema especialista.

\title{
SUMMARY: CRYSTALLOGRAPHY AND MINERALOGY OF THE CLAY FRACTION OF HARDSETTING HORIZONS IN SOILS OF COASTAL TABLELANDS IN BRAZIL
}

\begin{abstract}
The cohesion of hardsetting horizons of Brazilian soils has been mainly associated to inter-related physical and chemical factors, but an influence of mineralogical and crystallographic properties of clay minerals was also suggested by some researchers. In this study we tested the possibility that clay fraction of hardsetting horizons of soils from the Barreiras sediments of Coastal Tablelands are predominantly kaolinitic and highly crystalline and organized, which can favor a face-to-face arrangement of the crystals. The purpose of this study was to evaluate the crystallographic and mineralogical characteristics of clay minerals in hardsetting soil horizons from the sediment group Barreiras and their contribution to the hardsetting character. One non-hardsetting and five hardsetting horizons were studied along the coastal tableland. A kaolinite sample with high crystallinity degree was also included as reference. All profiles were analyzed for taxonomic classification and location of the horizons of interest. Organic matter and oxides were removed from the samples of the selected horizons. After soil dispersion, the clay fraction was individualized, treated and examined by X-ray diffraction (XRD) to determine the minerals and structure degree of order/disorder, according to the method described by Plançon \& Zacarie (1990). The degree of structural organization of the kaolinites of the hardsetting and non-hardsetting horizons was similar to and lower than the kaolinite used as reference. Results indicated that the hardsetting behavior of the studied soils could not be explained by clay packaging.
\end{abstract}

Index terms: kaolinite, compact horizons, crystallographic properties, expert system.

\section{INTRODUÇÃO}

Os sedimentos do Grupo Barreiras ocupam grande parte da faixa litorânea da Costa Oriental e Setentrional do Brasil (Bigarella, 1975) e, sobre eles, desenvolvem-se solos que apresentam horizontes com acentuada coesão entre as partículas (Giarola, 2002; Giarola, et al., 2003), por razões não esclarecidas. A coesão dos horizontes vem sendo associada a fatores múltiplos e inter-relacionados, principalmente de ordem química e física (Franzmeier et al., 1996; Mullins, 1999; Giarola et al., 2003). Em solos coesos brasileiros, a influência das propriedades mineralógicas e cristalográficas dos minerais da fração argila também já foi aventada por alguns pesquisadores (Resende et al., 2002).

Os sedimentos do Grupo Barreiras apresentam baixos teores de Fe e são essencialmente cauliníticos, com esqueleto quartzoso mal selecionado (Melo et al., 2002a,b), o que pode favorecer o adensamento dos solos deles originados (Zangrande, 1985; Arcanjo, 1990). Os horizontes adensados (coesos) apresentam grãos de quartzo dominando o esqueleto, envoltos por um plasma caulinítico denso e contínuo, com pouca tendência ao desenvolvimento de microestrutura (Giarola et al., 2001; Giarola, 2002; Lima et al., 2006).

O modelo caulinítico normalmente implica em desenvolvimento de uma macroestrutura do tipo de bloco e pode originar solos com densidade mais elevada, maior proporção de poros pequenos e menor permeabilidade, em razão do ajuste face a face das placas dos minerais da fração argila (Ferreira et al., 1999a). A ausência e a pouca expressão de óxidos de $\mathrm{Al}$ e $\mathrm{Fe}$ (gibbsita, goethita e hematita) e matéria orgânica, nesta ordem, podem favorecer a acomodação ordenada das placas de caulinita em escala microscópica, ao passo que o maior teor daqueles constituintes corresponde a um maior grau de desorganização e menor coesão (Resende et al., 2002). A justaposição ordenada das caulinitas é facilitada pela presença de cristais com alto grau de ordem estrutural e formas hexagonais (Dixon, 1989; Ferreira et al., $1999 b)$, características que podem ser associadas à ocorrência do caráter coeso em solos desenvolvidos de sedimentos do Grupo Barreiras. 
Plançon \& Zacharie (1990) propuseram um método para análise do grau de ordem/desordem estrutural das caulinitas. Este método permite avaliar defeitos relacionados às camadas com vacância em sítio octaédrico (Wc), defeitos de empilhamento entre camadas adjacentes devido às pequenas $(\delta)$ e grandes translações $(\mathrm{P})$, além de estimar o desenvolvimento de camadas ao longo do eixo $c(\mathrm{M})$ (Vieira-Coelho et al., 2000). Com o Método Especialista de Plançon e Zacharie - tradução livre de "expert system" - (ME), podem-se realizar análises quantitativas de diferentes características cristalográficas e defeitos estruturais (Plançon et al., 1988; Plançon \& Zacharie, 1990).

Neste trabalho, testou-se a hipótese de que a fração argila de horizontes coesos provenientes de solos desenvolvidos a partir de sedimentos do Grupo Barreiras é dominantemente caulinítica e apresenta elevado grau de cristalinidade e ordenamento, o que pode favorecer o ajuste face a face dos cristais. O objetivo deste trabalho foi avaliar as características mineralógicas e cristalográficas dos minerais da fração argila de horizontes coesos de solos formados em sedimentos do Grupo Barreiras e uma possível contribuição para a ocorrência do caráter coeso.

\section{MATERIAL E MÉTODOS}

\section{Amostragem}

Foram selecionados cinco horizontes coesos ( $\mathrm{P} 1 \mathrm{a}$ P5) e um não-coeso (P6), coletados em solos desenvolvidos sobre sedimentos do Grupo Barreiras, localizados nos municípios de Aracruz (Espírito Santo) (P1), Campos dos Goytacazes (Rio de Janeiro) (P2), Porto Seguro (Bahia) (P3), Cruz das Almas (Bahia) (P4) e Pacajus (Ceará) (P5 e P6) (Figura 1). O relevo das áreas amostradas compreende tabuleiros de topos aplainados, com altitudes de 20 a $200 \mathrm{~m}$, entalhados por vales estreitos e de fundo chato (Mabesoone, 1966).

\section{Análises para caracterização e classificação dos solos}

Os perfis de solo foram morfologicamente descritos segundo Santos et al. (2005). Para caracterização e classificação, amostras de solo coletadas de todos os horizontes dos perfis foram submetidas a análises químicas $(\mathrm{pH}$, teores trocáveis de $\mathrm{Ca}, \mathrm{Mg}, \mathrm{K}, \mathrm{Al}$ e C orgânico, óxidos extraídos por $\mathrm{H}_{2} \mathrm{SO}_{4}$ 1:1) e granulométricas (Embrapa, 1997, 2006) (Quadro 1)

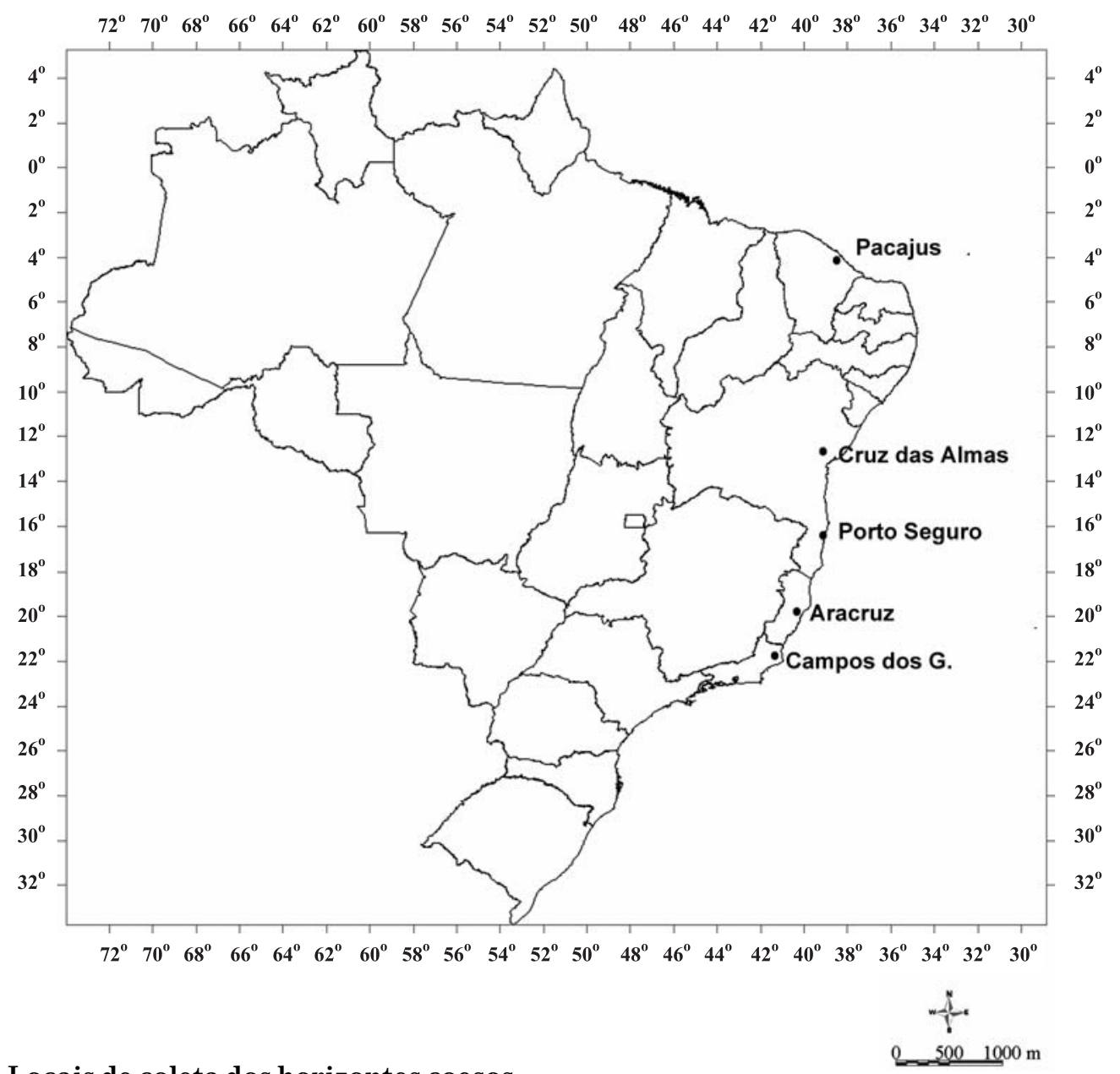

Figura 1. Locais de coleta dos horizontes coesos. 
Quadro 1. Atributos morfológicos e análise textural dos horizontes coesos amostrados em solos desenvolvidos em sedimentos do Grupo Barreiras

\begin{tabular}{|c|c|c|c|c|c|c|c|c|c|}
\hline Perfil & $\begin{array}{l}\text { Classe } \\
\text { de solo }\end{array}$ & Local & Horiz. $^{(1)}$ & $\begin{array}{l}\text { Estrutura } \\
\text { (solo seco) }\end{array}$ & Prof. $^{(2)}$ & $\begin{array}{l}\text { Argila } \\
<0,002\end{array}$ & $\begin{array}{c}\text { Silte } \\
0,002-0,05\end{array}$ & $\begin{array}{c}\text { Areia } \\
0,05-2,00\end{array}$ & $\begin{array}{c}\text { Classificação } \\
\text { textural }\end{array}$ \\
\hline & & & & & $\mathrm{m}$ & 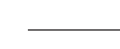 & $-\mathrm{g} \mathrm{kg}^{-1}$ & - & \\
\hline $\mathrm{P} 1$ & $\begin{array}{l}\text { Argissolo } \\
\text { Amarelo }\end{array}$ & Aracruz & Bt1 & maciça coesa & $0,39-0,88$ & 430 & 40 & 530 & Argilo-arenosa \\
\hline $\mathrm{P} 2$ & $\begin{array}{l}\text { Argissolo } \\
\text { Amarelo }\end{array}$ & Campos & $\mathrm{Bt}$ & maciça coesa & $0,60-0,98$ & 460 & 30 & 510 & Argilo-arenosa \\
\hline P3 & $\begin{array}{l}\text { Argissolo } \\
\text { Amarelo }\end{array}$ & $\begin{array}{l}\text { Porto } \\
\text { Seguro }\end{array}$ & Bt1 & maciça coesa & $0,38-0,55$ & 470 & 20 & 510 & Argilo-arenosa \\
\hline $\mathrm{P} 4$ & $\begin{array}{l}\text { Latossolo } \\
\text { Amarelo }\end{array}$ & $\begin{array}{l}\text { Cruz das } \\
\text { Almas }\end{array}$ & $\mathrm{AB} 1$ & $\begin{array}{l}\text { maciça coesa } \\
\text { a blocos }\end{array}$ & $0,21-0,38$ & 350 & 30 & 620 & $\begin{array}{l}\text { Franco-argilo- } \\
\text { arenosa }\end{array}$ \\
\hline P5 & $\begin{array}{l}\text { Argissolo } \\
\text { Acinzentado }\end{array}$ & Pacajus & Bt1 & maciça coesa & $0,97-1,20$ & 290 & 40 & 670 & $\begin{array}{l}\text { Franco-argilo- } \\
\text { arenosa }\end{array}$ \\
\hline $\mathrm{P} 6$ & $\begin{array}{l}\text { Argissolo } \\
\text { Amarelo }\end{array}$ & Pacajus & Bt1 & $\begin{array}{l}\text { maciça a } \\
\text { blocos }\end{array}$ & $1,30-1,72$ & 280 & 20 & 700 & $\begin{array}{l}\text { Franco-argilo- } \\
\text { arenosa }\end{array}$ \\
\hline
\end{tabular}

${ }^{(1)}$ Horiz.: horizonte do perfil. ${ }^{(2)}$ Prof.: profundidade inicial de coleta.

\section{Remoção de matéria orgânica, óxidos e dispersão de partículas}

Para remover a matéria orgânica, amostras dos horizontes coesos (Terra Fina Seca ao Ar - TFSA) foram tratadas com hipoclorito de sódio. Na sequência, efetuou-se uma extração com oxalato de amônio (em ausência de luz) para remoção de óxidos menos cristalinos (McKeague, 1978). No final, foram realizadas mais quatro extrações sucessivas com ditionito-citrato-bicarbonato, para remoção de óxidos mais cristalinos (Mehra \& Jackson, 1960).

As partículas da porção inorgânica foram dispersas por meio de solução de hexametafosfato de sódio (5\%, p/v), para análise granulométrica da TFSA e separação da fração argila para posterior análise química e mineralógica. A fração areia foi separada por tamisação com peneira com malha de $53 \mu \mathrm{m}$, e as frações argila e silte foram separadas por sedimentação com base na lei de Stokes (Gee \& Or, 2002). Uma amostra de fração argila natural (sem pré-tratamento) foi isolada para a determinação dos minerais.

\section{Análises químicas e mineralógicas da fração argila}

A composição química dos óxidos de Fe menos cristalinos de cada horizonte coeso foi avaliada em triplicatas de amostras, saturadas com $\mathrm{Na}$ e submetidas a uma extração com oxalato de amônio 0,2 $\mathrm{mol} \mathrm{L}^{-1}$, pH 3,0, em ausência de luz (McKeague, 1978). Após a extração, a suspensão foi centrifugada e os extratos acondicionados para avaliar os teores de $\mathrm{Fe}, \mathrm{Al}$ e Si. A dosagem dos elementos foi feita em espectrofotômetro de emissão por plasma (ICP-AES).

Os minerais da fração argila natural e tratada (moídas em almofariz e tamisadas em peneira de
$53 \mu \mathrm{m}$ ) foram analisados por difração de raios X (DRX) num difratômetro com goniômetro vertical Philips PW 3730, controlado por computador, monocromador de grafite para eliminação da radiação $K \beta$, radiação $\mathrm{CuK} \alpha(0,154186 \mathrm{~nm})$ e operado a uma potência de $40 \mathrm{kV}$ e corrente de $40 \mathrm{~mA}$. Para a identificação dos minerais utilizou-se $1 \mathrm{~s}$ de acumulação por passo a cada $0,02^{\circ}(2 \theta)$, na extensão 5 a $40^{\circ}$. Para análise do grau de desordem estrutural das caulinitas, utilizaram-se $10 \mathrm{~s}$ por passo em extensão similar à identificação dos minerais. Os dados obtidos pela DRX foram analisados no programa "Origin", com o qual também foram quantificadas as propriedades cristalográficas das caulinitas, segundo o Método Especialista de Plançon e Zacharie - tradução livre de expert system - (ME) (Plançon \& Zacharie, 1990). Para efeito de comparação dos resultados, uma amostra de caulinita de elevado grau de ordenamento estrutural (Montes et al., 2002) e outra proveniente de um horizonte não-coeso (Lima, 2004) foram utilizadas como referência.

\section{RESULTADOS E DISCUSSÃO}

De modo geral, a capacidade de troca de cátions em pH 7,0 (T) apresentou-se baixa, sempre inferior a $5 \mathrm{cmol}_{\mathrm{c}} \mathrm{kg}^{-1}$, refletindo a baixa disponibilidade de nutrientes e a mineralogia caulinítica dos solos (Quadro 2). Níveis semelhantes foram encontrados por Giarola (2002) e Lima (2004) em solos com comportamento similar.

A relação sílica/alumina (Ki) superior a 1,7 e os baixos teores de Fe extraídos por ataque sulfúrico (Quadro 2) estão dentro da faixa de caracterização de 
Quadro 2. Atributos químicos dos horizontes coesos

\begin{tabular}{|c|c|c|c|c|c|c|}
\hline Perfil de solo & $\mathbf{C}$ & $\mathbf{T}$ & $\mathrm{SiO}_{2}$ & $\mathrm{Al}_{2} \mathrm{O}_{3}$ & $\mathrm{Fe}_{2} \mathrm{O}_{3}$ & $\mathbf{K i}^{(1)}$ \\
\hline & $\mathrm{g} \mathrm{kg}^{-1}$ & $\mathrm{cmol}_{\mathrm{c}} \mathrm{kg}^{-1}$ & 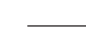 & $-\mathrm{g} \mathrm{kg}^{-1}$ & - & \\
\hline Aracruz (P1) & 25,99 & 4,0 & 270 & 250 & 40 & 1,8 \\
\hline Campos (P2) & 21,35 & 3,1 & 300 & 270 & 40 & 1,9 \\
\hline Porto Seguro (P3) & 5,22 & 3,1 & 110 & 150 & 30 & 1,3 \\
\hline Cruz das Almas (P4) & 22,56 & 4,4 & 260 & 220 & 50 & 2,0 \\
\hline Pacajus (P5) & 9,98 & 2,5 & 270 & 230 & 20 & 2,0 \\
\hline
\end{tabular}

(1) $\mathrm{Ki}=(\%) \mathrm{SiO}_{2} \times 1,7 /(\%) \mathrm{Al}_{2} \mathrm{O}_{3}$.

horizontes coesos (Embrapa, 1999). Os baixos teores de matéria orgânica (MO) e de óxidos de Fe (Quadro 2) podem favorecer o desenvolvimento de horizontes adensados. De acordo com Resende et al. (2002), a presença limitada destes tende a favorecer o maior ordenamento microestrutural das partículas e, por consequência, o aumento da coesão.

\section{Características químicas e mineralógicas da fração argila}

O material extraído pelo oxalato de amônio foi dominado principalmente por óxidos de alumínio menos cristalinos (Quadro 3). Em virtude das baixas concentrações de $\mathrm{Fe}$ nos sedimentos, os teores de $\mathrm{Fe}_{2} \mathrm{O}_{3}$ apresentaram-se muito baixos, assim como os teores de $\mathrm{SiO}_{2}$. Esses resultados corroboraram aqueles encontrados por Boulet et al. (1998), Moreau (2001), Melo et al. (2002a,b) em horizontes coesos de diferentes solos dos sedimentos do Grupo Barreiras.

Os teores de $\mathrm{Al}_{2} \mathrm{O}_{3}$ menos cristalinos foram superiores aos dos demais óxidos e similares entre os horizontes coesos de Aracruz, Campos, Porto Seguro e Pacajus. Apenas o perfil de Cruz das Almas apresentou teores significativamente superiores aos demais, alcançando praticamente o triplo dos teores determinados nos demais horizontes (Quadro 3).

Os teores de óxidos de Si também foram significativamente superiores no horizonte coeso de Cruz das Almas (Quadro 3). Neste horizonte, os teores de Si menos cristalinos apresentaram-se cerca de quatro vezes superiores aos demais. Embora o $\mathrm{SiO}_{2}$ ocorra em menor proporção, pequeno teor desse constituinte pode contribuir com uma coesão temporária maior das partículas, deixando o material duro a extremamente duro quando seco (Drees et al., 1989). Essa maior coesão pode estar relacionada com a formação de pontes de sílica, descritas pelos modelos de deposição de Chadwick et al. (1987) e Franzmeier et al. (1989), propostos para horizontes endurecidos.

Os resultados da difratometria de raios $\mathrm{X}$ da fração argila (Figura 2) demonstraram que a mineralogia dos horizontes coesos é predominantemente caulinítica, similar a estudos anteriores realizados por Melo et al. (2002), Giarola (2002) e Lima (2004).
Quadro 3. Teores médios $(n=3)$ dos elementos extraídos pelo oxalato de amônio da fração argila dos horizontes coesos (Tukey, 5 \%)

\begin{tabular}{llll}
\hline \multicolumn{1}{c}{$\begin{array}{c}\text { Local de } \\
\text { amostragem }\end{array}$} & $\mathrm{Al}_{2} \mathbf{O}_{3}$ & $\mathbf{S i O}_{2}$ & $\mathbf{F e}_{2} \mathbf{O}_{3}$ \\
& & & \\
\cline { 2 - 4 } & & $\mathrm{g} \mathrm{kg}^{-1}$ & \\
\cline { 2 - 4 } Aracruz (P1) & $1,12 \mathrm{~b}$ & $0,20 \mathrm{c}$ & $0,73 \mathrm{ab}$ \\
Campos (P2) & $1,84 \mathrm{~b}$ & $0,47 \mathrm{~b}$ & $0,66 \mathrm{abc}$ \\
Porto Seguro (P3) & $1,65 \mathrm{~b}$ & $0,36 \mathrm{bc}$ & $0,55 \mathrm{bc}$ \\
Cruz das Almas (P4) & $3,87 \mathrm{a}$ & $0,74 \mathrm{a}$ & $0,98 \mathrm{a}$ \\
Pacajus (P5) & $1,25 \mathrm{~b}$ & $0,24 \mathrm{c}$ & $0,13 \mathrm{~d}$ \\
\hline
\end{tabular}

A composição mineralógica observada é comum à fração argila dos solos dos sedimentos do Grupo Barreiras, em função do intenso intemperismo por que passou o material parental, bem como pelas condições climáticas e geomorfológicas citadas por UFV (1984) e Ribeiro (1996), que favoreceram a hidrólise e remoção inicial dos óxidos de Fe e a posterior concentração de caulinita na fração argila (Melo et al., 2001).

\section{Características cristalográficas das caulinitas}

Os resultados da aplicação do Método Especialista (Quadro 4) demonstraram que as caulinitas dos horizontes coesos analisados são constituídas por uma única fase, o que possibilitou a análise das propriedades para determinação do grau de ordem/ desordem estrutural dos cristais. Observa-se que os valores médios de $M$ - que estima o desenvolvimento de camadas ao longo do eixo $c$ - apresentaram diferenças significativas entre os horizontes. Os horizontes coesos e o não-coeso foram similares entre si, porém inferiores ao da amostra de referência (JA 19). Esses resultados são indicativos de que as lâminas das caulinitas dos horizontes coesos (P1 a P5) e nãocoeso (P6) apresentaram menor grau de desenvolvimento das camadas ao longo do eixo $c$ quando comparadas às caulinitas bem ordenadas (amostra JA 19). 
Os valores médios estimados para $W c$ - que representa a proporção de defeitos referentes aos sítios vacantes na folha octaédrica das caulinitas - foram similares entre os horizontes coesos (P2 a P5), nãocoeso (P6) e a testemunha (JA 19) (Quadro 4), com exceção do horizonte coeso de Aracruz (P1). Embora as diferenças para a maioria dos horizontes coesos não tenham sido significativas, os valores pouco superiores de $W c$ em relação à amostra de referência indicaram maior proporção de defeitos.

Os índices médios das propriedades referentes aos defeitos de empilhamento relacionados às pequenas translações $(\delta)$ e às grandes translações $(P)$ não apresentaram diferenças entre os horizontes coesos (Quadro 4), mas foram significativamente superiores ao da testemunha. Os resultados indicaram que tanto as caulinitas dos horizontes coesos (P1 a P5) como as do não-coeso (P6) apresentaram grau de ordenamento estrutural similar, porém menor do que o da caulinita de referência (JA 19). Se o grau de cristalinidade elevado fosse responsável pelo ajuste face a face dos cristais de caulinita e, por sua vez, da manifestação do caráter coeso, então os horizontes coesos (P1 a P5) deveriam apresentar propriedades diferentes daquelas do horizonte não-coeso (P6) e mais próximas da caulinita de referência. Embora não haja trabalhos que tenham aplicado o Método Especialista de Plançon \& Zacharie (1990) para avaliar as características cristalográficas (propriedades indicativas de ordem e desordem estrutural) de minerais da fração argila em solos do Brasil, Melo et al. (2002a) observaram maior ordenamento estrutural das caulinitas em Argissolo Amarelo coeso, por meio do índice de cristalinidade de Hughes e Brown (Hughes \& Brown, 1979).

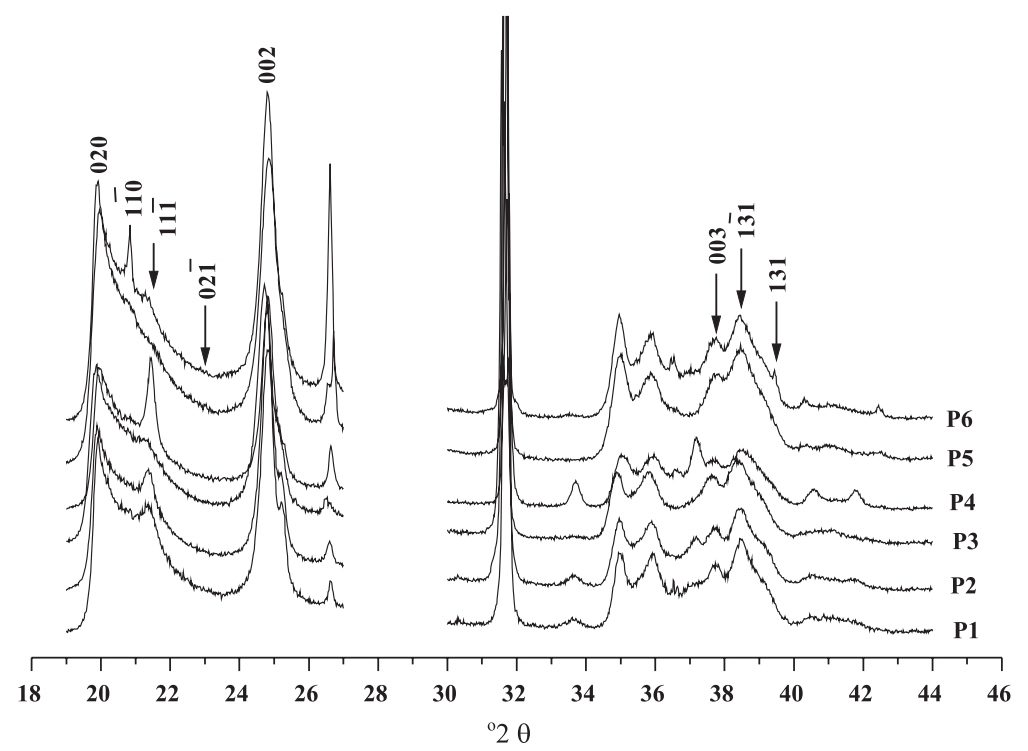

Figura 2. Difratogramas de raios X da fração argila de horizontes coesos (P1 a P5) e não-coeso (P6) de solos provenientes dos sedimentos do Grupo Barreiras.

Quadro 4. Propriedades obtidas da análise do grau de ordem/desordem estrutural das caulinitas de horizontes coesos (P1 a P5) e não-coeso (P6) de solos dos sedimentos do Grupo Barreiras e da caulinita de referência (JA 19), conforme o Sistema Especialista de Plançon \& Zacharie (1990) ${ }^{(1)}$

\begin{tabular}{|c|c|c|c|c|c|}
\hline \multirow{2}{*}{ Local de amostragem } & \multirow{2}{*}{ Número de fases } & \multicolumn{4}{|c|}{ Propriedade do sistema especialista } \\
\hline & & $M^{(1)}$ & $W c^{(2)}$ & $\delta^{(3)}$ & $P^{(4)}$ \\
\hline Aracruz (P1) & 1 & $31 \mathrm{~cd}$ & $0,15 \mathrm{a}$ & $0,06 \mathrm{a}$ & $0,40 \mathrm{a}$ \\
\hline Campos (P2) & 1 & $33 \mathrm{bc}$ & $0,06 \mathrm{ab}$ & $0,06 \mathrm{a}$ & $0,40 \mathrm{a}$ \\
\hline Porto Seguro (P3) & 1 & $30 \mathrm{~d}$ & $0,09 \mathrm{ab}$ & $0,06 \mathrm{a}$ & $0,40 \mathrm{a}$ \\
\hline Cruz das Almas (P4) & 1 & $33 \mathrm{~b}$ & $0,05 \mathrm{ab}$ & $0,06 \mathrm{a}$ & $0,40 \mathrm{a}$ \\
\hline Pacajus (P5) & 1 & $26 \mathrm{de}$ & $0,02 \mathrm{~b}$ & $0,06 \mathrm{a}$ & $0,40 \mathrm{a}$ \\
\hline Pacajus (P6) ${ }^{(5)}$ & 1 & $28 \mathrm{de}$ & $0,01 \mathrm{~b}$ & $0,06 \mathrm{a}$ & $0,39 \mathrm{a}$ \\
\hline Caulinita JA $19^{(6)}$ & 1 & $51 \mathrm{a}$ & $0,00 \mathrm{~b}$ & $0,04 \mathrm{~b}$ & $0,35 \mathrm{~b}$ \\
\hline
\end{tabular}

(1) $M$, índice de desenvolvimento das camadas ao longo do eixo $c{ }^{(2)} W c$, índice dos defeitos relacionados às camadas com vacância em sítios octaédricos. ${ }^{(3)} \delta$, índice dos defeitos de empilhamento relacionados às pequenas translações. ${ }^{(4)} P$, índice dos defeitos de empilhamento relacionados às grandes translações. ${ }^{(5)}$ Amostra de caulinita de horizonte não-coeso utilizada como referência.

(6) Amostra de caulinita de alto grau de ordenamento utilizada como referência. 


\section{CONCLUSÃO}

Os horizontes coesos avaliados apresentaram a fração argila dominada por caulinitas com grau de ordenamento estrutural elevado e baixos teores de óxidos cristalinos e pouco cristalinos. Todos os horizontes coesos apresentaram caulinita com grau de ordenamento estrutural similar ao do horizonte nãocoeso e inferior ao da caulinita de referência, o que não permitiu associar o empacotamento da fração argila com a manifestação do caráter coeso nos horizontes estudados a partir das propriedades obtidas pelo Método Especialista de Plançon e Zacharie.

\section{LITERATURA CITADA}

ARCANJO, J.D. La formation Barreiras dans la region de Vitoria, ES. Strasbourg, Université Louis Pasteur, 1990. 110p. (Tese de Doutorado)

BIGARELA, J.J. The Barreiras Group in Northeastern Brazil. An. Acad. Bras. Ci., 47:365-393, 1975

BOULET, R.; FRITSCH, E.; FILIZOLA, H.F.; ARAÚJO FILHO, J.C.; LEPRUN, J.C.; BARRETO, F.; BALAN, E. \& TESSIER, D. Iron bands, fragipans and duripans in the Northeaster plateaus of Brazil - properties and genesis. Canadian J. Soil Sci., 78:519-530, 1998.

CHADWICK, O.A.; HENDRICKS, D.M. \& NETTLETON, W.D. Silica in duric soils: I. A depositional model. Soil Sci. Soc. Am. J., 51:975-982, 1987.

DIXON, J.B. Kaolin and serpentine group minerals. In: DIXON, J.B. \& WEED, S.B., eds. Minerals in soil environments. 2.ed. Madison, Soil Science Society of America, 1989. p.467-525.

DREES, L.R.; WILDING, L.P. \& SMECK, N.E. Silica in soils: Quartz and disordered silica polymorphs. In: DIXON, J.B. \& WEED, S.B., eds. Minerals in soil environments. 2.ed. Madison, Soil Science Society of America,, 1989. p.913974 .

EMPRESA BRASILEIRA DE PESQUISA AGROPECUÁRIA EMBRAPA. Centro Nacional de Pesquisa de Solos. Sistema brasileiro de classificação de solos. 2.ed. Rio de Janeiro, 2006. 306p.

EMPRESA BRASILEIRA DE PESQUISA AGROPECUÁRIA EMBRAPA. Centro Nacional de Pesquisa de Solos. Manual de métodos de análise de solo. 2.ed. Rio de Janeiro, 1997. $212 p$.

FERREIRA, M.M.; FERNANDES, B. \& CURI, N. Influência da mineralogia da fração argila nas propriedades físicas de Latossolos da Região Sudeste do Brasil. R. Bras. Ci. Solo, 23:515-524, 1999b.

FERREIRA, M.M.; FERNANDES, B. \& CURI, N. Mineralogia da fração argila e estrutura de Latossolos da Região Sudeste do Brasil. R. Bras. Ci. Solo, 23:507514, 1999a.
FRANZMEIER, D.P.; CHARTRES, C.J. \& WOOD, J.T. Hardsetting soils in Southeast Australia: Landscape and profile processes. Soil Sci. Soc. Am. J., 60:1178-1187, 1996.

FRANZMEIER, D.P.; NORTON, L.D. \& STEINHARDT, G.C. Fragipan formation in Midwestern United States. In: SMECK, N.E. \& CIOLKOSZ, E.J. Fragipans: Their occurrence, classification and genesis. Madison, Soil Science Society of America, 1989. p.69-97. (Special Publication, 24)

GEE, G.W. \& OR, D. Particle-size analysis. In: DANE, J.H. \& TOPP, G.C., eds. Methods of soil analysis. Physical methods. Madison, Soil Science Society of America, 2002. Part 4. p.255-293.

GIAROLA, N.F.B.; SILVA, A.P.; IMHOFF, S. \& DEXTER, A.R. Contribution of natural soil compaction on hardsetting behavior. Geoderma, 113:95-108, 2003.

GIAROLA, N.F.B. Similaridades entre solos coesos e hardsetting: Caracterização do comportamento físico. Piracicaba, Escola Superior de Agricultura Luiz de Queiroz, 2002. 65p. (Tese de Doutorado)

GIAROLA, N.F.B.; SILVA, A.P.; TORMENA, C.A.; SOUZA, L.S. \& RIBEIRO, L.P. Similaridades entre o caráter coeso dos solos e o comportamento hardsetting: Estudo de caso. R. Bras. Ci. Solo, 25:239-247, 2001.

HUGHES, J.C. \& BROWN, G. A crystallinity indexd for soil kaolins and its relation to parent rock, climate and soil maturity. J. Soil. Sci., 30:557-563, 1979.

LIMA, H.V.; SILVA, A.P.; SANTOS, M.C.; COOPER, M. \& ROMERO, R.E. Micromorphology and image analysis of a hardsetting Ultisol (Argissolo) in the State of Ceará (Brazil). Geoderma, 132:416-426, 2006.

LIMA, H.V. Identificação e caracterização do comportamento físico de solos coesos no Estado do Ceará. Piracicaba, Escola Superior de Agricultura Luiz de Queiroz, 2004. 85p. (Tese de Doutorado)

MABESOONE, J.M. Relief of Northeastern Brazil and its correlated sediments. Zeitschrift Geom., 4:419-453, 1966.

McKEAGUE, J.A. Manual on soil sampling and methods of analysis. Ottawa, Canadian Society of the Soil Science, 1978. 212p.

MEHRA, O.P. \& JACKSON, M.L. Iron oxide removal from soils and clay by a dithionite-citrate system buffered with sodium bicarbonate. Clays Clay Miner., 7:317-327, 1960.

MELO, V.F.; SCHAEFER, C.E.G.R.; SINGH, B.; NOVAIS, R.F. \& FONTES, M.P.F. Propriedades químicas e cristalográficas da caulinita e dos óxidos de ferro em sedimentos do Grupo Barreiras no município de Aracruz, estado do Espírito Santo. R. Bras. Ci. Solo, 26:53-64, $2002 \mathrm{a}$. 
MELO, V.F.; NOVAIS, R.F.; SCHAEFER, C.E.G.R.; FONTES, M.P.F. \& SINGH, B. Mineralogia das frações areia, silte e argila de sedimentos do Grupo Barreiras no município de Aracruz, estado do Espírito Santo. R. Bras. Ci. Solo, 26:29$41,2002 \mathrm{~b}$.

MELO, V.F.; SINGH, B.; SCHAEFER, C.E.G.R.; NOVAIS, R.F. \& FONTES, M.P.F. Chemical and mineralogical properties of kaolinite-rich Brazilian soils. Soil Sci. Soc. Am. J., 65:1324-1334, 2001.

MONTES, C.R.; MELFI, A.J.; CARVAlHO, A.; VIEIRACOELHO, A.C. \& FORMOSO, M.L.L. Gênesis, mineralogy and geochemistry of kaolin deposits of Jari River, Amapá State, Brazil. Clay Miner., 50:497-506, 2002 .

MOREAU, A.M.S.S. Gênese, mineralogia e micromorfologia de horizontes coesos, fragipã e duripã em solos dos Tabuleiros Costeiros do Sul da Bahia. Viçosa, MG, Universidade Federal de Viçosa, 2001. 138p. (Tese de Doutorado)

MULLINS, C.E. Hardsetting soils. In: SUMNER, M.E., ed. Handbook of soil science. New York, CRC Press, 1999. p.G65-G87.

PLANÇON, A. \& ZACHARIE, C. An expert system for the structural characterization of kaolinites. Clay Miner., 25:249-260, 1990.

PLANÇON, A.; GIESE, R.F.; SNYDER, R. The Hinckley index for kaolinites. Clay Miner., 23:249-260, 1988.
RESENDE, M.; CURI, N.; REZENDE, S.B. \& CORRÊA, S.A. Pedologia: Base para distinção de ambientes. 4.ed. Viçosa, MG, NEPUT, 2002. 338p.

RIBEIRO, L.P. Gênese, evolução e degradação dos solos amarelos coesos dos Tabuleiros Costeiros. In: REUNIÃO TÉCNICA SOBRE SOLOS COESOS DOS TABULEIROS COSTEIROS, Cruz das Almas, 1996. Anais. Aracaju, Embrapa, CPATC; CNPMF; EAUFBA; IGUFBA, 1996. p.27-35.

SANTOS, R.D.; LEMOS, R.C.; SANTOS, H.G.; KER, J.C. \& ANJOS, L.H.C. Manual de descrição e coleta de solos no campo. 5.ed. Viçosa, MG, Sociedade Brasileira de Ciência do Solo, 2005. 100p.

UNIVERSIDADE FEDERAL DE VICOSA-UFV. Caracterização de solos e avaliação dos principais sistemas de manejo dos Tabuleiros Costeiros do Baixo Rio Doce e das Regiões Norte do Estado do Espírito Santo e sua interpretação para uso agrícola. Viçosa, MG, 1984. 153p.

VIEIRA-COELHO, A.C.; MONTES, C.R. \& VALERA, T.S. Estudo do grau de ordem/desordem estrutural de caulinitas em caulins da Região Amazônica por difratometria de raios X. In: CONGRESSO BRASILEIRO DE CERÂMICA, 44., São Pedro, 2000. Anais. São Pedro, 2000. p.201-213.

ZANGRANDE, M.B. Caracterização e interpretação para uso de um Podzólico Vermelho-Amarelo abrúptico dos platôs litorâneos no norte do Espírito Santo. Viçosa, MG, Universidade Federal de Viçosa, 1985. 81p. (Tese de Mestrado) 